\section{SOUND TRANSMISSION IN THE OCEAN}

Introduction to the Theory of Sound Transmission with Application to the Ocean

By C. B. Officer. (McGraw-Hill Series in the Geological Sciences.) Pp. viii +284. (London: MeGraw-Hill Publishing Company, Ltd., 1958.) 77s. $6 d$.

7 THE author of this volume is associate professor in the Department of Geology, The Rice Institute, and associate in geophysics, Woods Hole Oceanographic Institution. These facts are mentioned to indicate that the book is of interest not only to physicists who are concerned with sound propagation in the sea, but also to geologists and geophysicists who are concerned with the wider aspects of the subject.

The propagation of sound in the ocean, in shallow seas, and in the Earth beneath the sea has important implications which concern a wide field of science, namely, physics, geophysics and oceanography.

In the preface the author directs attention to the fact that the preparation of the book has been sponsored ("encouraged and supported") by the Office of Naval Research and the U.S. Bureau of Ships, thus indicating its importance to those concerned with the applied aspects of a study of sound propagation in the ocean.

Reference is also made in the preface to many well. known American workers in this field, for example, J. B. Hersey, M. Ewing, J. L. Worzel and others.

The subject-matter of the book is divided into six chapters. The first of these deals with general wave theory for an elastic medium, including the cases of waves of finite amplitude and shock fronts. The second chapter is concerned with the 'ray' and 'normal-mode' theories of sound propagation, with refraction of a dispersive wave-train, and with prescribed boundary conditions. In Chapter 3, these considerations are applied to the case of transmission in shallow water where interference due to multiple surface and bottom images has to be taken into account. The fourth chapter deals with the case of deep oceanic water, where the bottom is not involved. In this case refraction due to temperature and pressure effects on sound-velocity is considered. Chapter 5 is concerned with 'reflectivity' of boundary layers, reflexion from three layers, and the interpretation of echo-sounding records and of geophysical reflexion and refraction profiles. The concluding chapter, "Attenuation", covers absorption in a viscous fluid, scattering and diffraction, and reception by a linear array.

Chapters 3 and 4, which deal respectively with the transmission in shallow water (inshore seas) and in deep water (tho ocoans), show the difficulties involved in attempting to arrive at a reliable theoretical solution of wave propagation in the sea. Consideration must ultimately be given to many factors, for example, the effects of depth and configuration of the sea-bed, the physical (acoustic) properties of the sea-bed material, the sound-velocity structure of the water (temperature- and pressure-gradients as they influence velocity of propagation), the shape of the sea-surface (waves and swell), the presence of soundscatterers in the water, and so on. As the author very truly states, "A complete formulation with all possible variations of the above environmental conditions would be very difficult to obtain." The theoretical complications in shallow water transmission due to multiple reflexions between seasurface and sea-bed are dealt with on both 'ray' and 'mode' theories, but there is clearly much more to be done to predict the sound-distribution in a shallow sea even under the most 'ideal' theoretical conditions of isothermal water and a flat bottom parallel to a flat sea surface.

The problem is a difficult one, and Prof. Officer has made a praiseworthy effort in presenting the theoretical aspect in a stimulating and readable fashion. As an introduction to the subject the book will serve a very useful purpose in arousing interest in physicists, geophysicists and oceanographers alike.

The book is well written, illustrated and produced and can be recommended to all those interested in this difficult but fascinating study of sound transmission in the sea.

A. B. WOOD

\section{THEORY OF HIGH EXPLOSIVES}

The Science of High Explosives

By Prof. Melvin A. Cook. (American Chemical Society Monograph Series, No. 139.) Pp. xv +440. (New York: Reinhold Publishing Corporation; London: Chapman and Hall, Ltd., 1958.) 180s. net.

THIS book is a valuable addition to the rather sparse literature on the subject of explosives. Its scope is wide; but, as the title suggests, it is concerned more with the basic physical and chemical principles underlying the behaviour of explosives than with practical applications. Nevertheless, there are parts that will be of general interest, in particular the chapters describing the modern explosives industry and the explosion properties and blasting action of high explosives.

Perhaps the heart of the book is formed by those chapters dealing with the mechanism of initiation and propagation of detonation and the accompanying phenomena. Particular attention is paid to the nature of the reaction zone in detonation waves, and the author considers that the classical treatments of Zeldovich, von Neumann, Doering, and others should be modified to include the notion of heat conduction. In addition to claiming that this modification gives a better explanation of the observed distribution of parameters in the reaction zone (for example, the evidence for the existence of a pressure 'spike' predicted by the original theories is inconclusive), he says that it explains what happens to the critical enthalpy that is necessary to initiate detonation. $\mathrm{H}_{\Theta}$ considers that this enthalpy is contained within the reaction-zone and propagated as a heat pulse. The author claims to have obtained photographic evidence of a heat pulse during the growth to detonation of an initially non-reactive shock-wave in the liquid explosive nitromethane, but unfortunately the features in the original colour photographs to which he a,scribes considerable significance do not show up well in the black-and-white reproductions. Also discussed in some detail are the respective theories of non-ideal detonation due to Jones and to Eyring and others. An alternative theory, called the geometrical model, is put forward which asserts that not only must lateral losses, which affect only the equations of continuity, be taken into account but also that only part of the reaction zone lies between the detonation front and the Chapman-Jouguet plane, so that not all the chemical energy released is available for support. 